\title{
A Direct Transformation of Aryl Aldehydes to Benzyl Iodides Via Reductive Iodination
}

\author{
Jayaraman Sembian Ruso, Nagappan Rajendiran*, and Rajendran Senthil Kumaran ${ }^{\dagger, *}$ \\ Department of Polymer Science, Maramalai Campus, University of Madras, Guindy, Chennai - 600025, India. \\ ${ }^{*}$ E-mail: nrajendiar@yahoo.com \\ ${ }^{\dagger}$ Syngene International Ltd, Biocon Park \#2 and 3, Bommasandra, Jigani Link Road, Bangalore-560099, India. \\ ${ }^{*}$ E-mail: senthilkr76@rediffmail.com
}

(Received March 13, 2013; Accepted December 17, 2013)

\begin{abstract}
A facile transformation of aryl aldehydes to benzyl iodides through one-pot reductive iodination is reported. This protocol displays remarkable functional group tolerance and the title compound was obtained in good to excellent yield.
\end{abstract}

Key words: Benzyl iodides, Triethylsilane, Reductive iodination

\section{INTRODUCTION}

Benzyl iodides are important subunit in organic synthesis. They are found to have broad applications in fields of fine chemicals, pharmaceuticals, medicinal chemistry and drug discovery. ${ }^{1-4}$ Although they possess numerous synthetic utilities, it has been primarily used to form carbon-carbon and carbon-heteroatom bonds. Unlike other halides, benzyl iodides are prepared freshly before use due to their low stability. Evidently, many synthetic protocols have been developed for the synthesis of benzyl iodides, however, most of the methods describe their preparation from the corresponding alcohol. ${ }^{5}$ Moreover, the projected benzyl iodide synthesis from aryl aldehyde involves two steps, proceeds via the intermediacy of benzyl alcohol. Conversely, it can be obtained in a single step by employing reductive iodination. ${ }^{6}$ Despite of its importance, this protocol has been less explored and thus found limited exploitation in synthesis.

Over the past few decades, organosilanes are emerged as popular reagents in synthetic chemistry. ${ }^{7}$ In the past, chlorotriethylsilane, dichloromethylsilane and polymethylhydrosiloxane were used for the reductive iodination, however, they had limited substrate scope. ${ }^{6 a-d}$ Also, few of these methods suffer from tedious work up procedures, harsh conditions and long reaction time. Triethylsilane $\left(\mathrm{Et}_{3} \mathrm{SiH}\right)$ is another versatile and commercially available reagent utilized for many transformations, for example, the reduction of carbonyl derivative. ${ }^{8}$ Consequently, we planned to develop one-pot strategy to access benzyl iodide from aryl aldehyde using triethylsilane. Herein, we disclose a facile and convenient reductive iodination of aryl aldehydes using $\mathrm{Et}_{3} \mathrm{SiH}$ and trifluoromethanesulfonic acid $(\mathrm{TfOH})$ in presence of sodium iodide.

\section{EXPERIMENTAL}

All reagents were purchased from commercial suppliers and were used without purification. Melting points were determined in Buchi B-545 melting point apparatus and were uncorrected. ${ }^{1} \mathrm{H}$ NMR and ${ }^{13} \mathrm{C}$ NMR spectra were recorded on a Bruker Advance and Varian $400 \& 300$ NMR MHz spectrometers in DMSO- $d_{6} \& \mathrm{CDCl}_{3}$ solution using TMS as an internal reference and ${ }^{13} \mathrm{C}$ NMR spectra were recorded on $100 \& 75 \mathrm{MHz}$. Mass spectra were recorded on GC-MS using 230-400 mesh silica gel.

\section{Typical Experimental Procedure for the Preparation of Benzyl Iodides}

To an ice-cold solution of 4-bromo benzaldehyde 1a (0.18 g, $1 \mathrm{mmol}$ ) in $\mathrm{CH}_{3} \mathrm{CN} / \mathrm{DME}$ (5 mL, 8:2) was added $\mathrm{NaI}(0.30 \mathrm{~g}, 2 \mathrm{mmol})$ and trifluoromethanesulphonic acid $(0.09 \mathrm{~mL}, 1 \mathrm{mmol})$. Triethysilane $(0.30 \mathrm{~mL}, 2 \mathrm{mmol})$ was slowly added to the mixture and allowed to stir at room temperature for $1 \mathrm{~h}$. The reaction was monitored by GC. The reaction was quenched with $10 \% \mathrm{NaHCO}_{3}$ solution $(10 \mathrm{~mL})$ on completion and the reaction mass was diluted with DCM $(50 \mathrm{~mL})$. The organic layer was separated, dried over anhydrous $\mathrm{Na}_{2} \mathrm{SO}_{4}$, evaporated and the crude mass was purified by silica gel flash column chromatography ( $2 \%$ ethyl acetate in petroleum ether) to give 4-bromo benzyl iodide $2 \mathbf{a}$ (266 $\mathrm{mg}, 90 \%$ ).

1-Bromo-4-(iodomethyl)benzene 2a

White solid; m.p. $71-73{ }^{\circ} \mathrm{C} ;{ }^{1} \mathrm{HNMR}\left(400 \mathrm{MHz}, \mathrm{CDCl}_{3}\right) \delta$ 
$7.42(\mathrm{~d}, J=7.9 \mathrm{~Hz}, 2 \mathrm{H}), 7.25(\mathrm{~d}, J=7.8,2 \mathrm{H}), 4.40(\mathrm{~s}, 2 \mathrm{H})$;

${ }^{13} \mathrm{C}$ NMR $\left(75 \mathrm{MHz}, \mathrm{CDCl}_{3}\right) \delta 138.3,131.9,130.3,121.6$,

4.3. GCMS: 296.

1-Bromo-2-(iodomethyl)benzene 2b

White solid; m.p. $58-60{ }^{\circ} \mathrm{C} ;{ }^{1} \mathrm{HNMR}\left(300 \mathrm{MHz}, \mathrm{CDCl}_{3}\right) \delta$ 7.55-7.52 (m, 1H), 7.46-7.43 (m, 1H), 7.29-7.23 (m, 1H), 7.15-7.10 (m, 1H), $4.5(\mathrm{~s}, 2 \mathrm{H}) ;{ }^{13} \mathrm{C} \mathrm{NMR}\left(75 \mathrm{MHz}, \mathrm{CDCl}_{3}\right)$ $\delta$ 138.3, 133.4, 130.5, 129.5, 127.9, 124.0, 5.6; GCMS: 296.

1-Bromo-3-(iodomethyl)benzene 2c

Pale yellow solid; m.p. $50-53{ }^{\circ} \mathrm{C} ;{ }^{1} \mathrm{H}$ NMR $(300 \mathrm{MHz}$, $\left.\mathrm{CDCl}_{3}\right) \delta 7.53(\mathrm{t}, J=1.7 \mathrm{~Hz}, 1 \mathrm{H}), 7.39-7.36(\mathrm{~m}, 1 \mathrm{H}), 7.29$ (t, $J=7.9 \mathrm{~Hz}, 1 \mathrm{H}), 7.17(\mathrm{t}, J=7.8 \mathrm{~Hz}, 1 \mathrm{H}), 4.39$ (s, 2H); ${ }^{13} \mathrm{C} \mathrm{NMR}\left(100 \mathrm{MHz}, \mathrm{CDCl}_{3}\right) \delta 141.4,1331.6,130.9,130.2$, 127.3, 122.4, 3.6; GCMS: 296.

1-Chloro-4-(iodomethyl)benzene 2d

Pale yellow solid; m.p. $62-64{ }^{\circ} \mathrm{C} ;{ }^{1} \mathrm{H}$ NMR $(400 \mathrm{MHz}$, $\left.\mathrm{CDCl}_{3}\right) \delta 7.33(\mathrm{~d}, J=2.2 \mathrm{~Hz}, 2 \mathrm{H}), 7.28(\mathrm{~d}, J=2.2 \mathrm{~Hz}, 2 \mathrm{H})$, 4.44 (s, 2H); $\left.{ }^{13} \mathrm{C} \mathrm{NMR} \mathrm{(75} \mathrm{MHz,} \mathrm{CDCl}_{3}\right) \delta 137.8,133.5$, 130.0, 128.9, 4.1; GCMS: 252.

1-Fluoro-2-(iodomethyl)benzene 2e

Yellow liquid; ${ }^{1} \mathrm{H} \mathrm{NMR}\left(300 \mathrm{MHz}, \mathrm{CDCl}_{3}\right) \delta 7.39-7.36$ (m, 1H), 7.34-7.23 (m, 1H), 7.11-6.99 (m, 2H), 4.45 (s, 2H); ${ }^{13} \mathrm{C}$ NMR $\left(75 \mathrm{MHz}, \mathrm{CDCl}_{3}\right) \delta 160.3,130.7,129.8,126.4$, 124.4, 115.8, -3.5; GCMS: 236.

2-Chloro-4-fluoro-1-(iodomethyl)benzene $2 \mathrm{f}$

White solid; m.p. $50-52{ }^{\circ} \mathrm{C} ;{ }^{1} \mathrm{H}$ NMR $\left(400 \mathrm{MHz}, \mathrm{CDCl}_{3}\right)$ $\delta$ 7.41-7.38 (m, 1H), 7.12-7.09 (m, 1H), 6.96-6.92 (m, 1H), $4.49(\mathrm{~s}, 2 \mathrm{H}) ;{ }^{13} \mathrm{C}$ NMR $\left(75 \mathrm{MHz}, \mathrm{CDCl}_{3}\right) \delta 161.9,134.5$, 132.8, 131.5, 117.4, 114.6, 1.2; GCMS: 270.

1-Bromo-2-fluoro-3-(iodomethyl)benzene 2g

White solid; m.p. $54-56{ }^{\circ} \mathrm{C}$; ${ }^{1} \mathrm{H}$ NMR $\left(400 \mathrm{MHz}, \mathrm{CDCl}_{3}\right)$ $\delta$ 7.27-7.21 (m, 3H), $4.38(\mathrm{~s}, 2 \mathrm{H}) ;{ }^{13} \mathrm{C}$ NMR $(75 \mathrm{MHz}$, $\left.\mathrm{CDCl}_{3}\right) \delta 159.9,131.6,127.8,125.9,122.2,119.5,-4.8$; GCMS: 314.

4-Bromo-2-(iodomethyl)-1-methoxybenzene $2 \mathrm{~h}$

Off-white solid; m.p. $55-58^{\circ} \mathrm{C} ;{ }^{1} \mathrm{HNMR}\left(400 \mathrm{MHz}, \mathrm{CDCl}_{3}\right)$ $\delta 7.42(\mathrm{~d}, J=2.4 \mathrm{~Hz}, 1 \mathrm{H}), 7.34(\mathrm{dd}, J=8.72,2.4 \mathrm{~Hz}, 1 \mathrm{H})$, 6.72-6.70 (d, $J=8.72 \mathrm{~Hz}, 1 \mathrm{H}), 4.40(\mathrm{~s}, 2 \mathrm{H}), 3.89(\mathrm{~s}, 3 \mathrm{H})$; ${ }^{13} \mathrm{C}$ NMR $\left(100 \mathrm{MHz}, \mathrm{CDCl}_{3}\right) \delta 156.2,132.6,132.1,129.6$, 112.7, 112.5, 55.8, -0.7; GCMS: 326.

1-Bromo-3-chloro-2-(iodomethyl)benzene $2 \mathrm{i}$

Off-white solid; m.p. $75-78^{\circ} \mathrm{C} ;{ }^{1} \mathrm{HNMR}\left(400 \mathrm{MHz}, \mathrm{CDCl}_{3}\right)$ $\delta 7.47(\mathrm{~d}, J=8.04 \mathrm{~Hz}, 1 \mathrm{H}), 7.33(\mathrm{~d}, J=8.04 \mathrm{~Hz}, 1 \mathrm{H}), 7.07$ (t, $J=8.04 \mathrm{~Hz}, 1 \mathrm{H}), 4.71(\mathrm{~s}, 2 \mathrm{H}) ;{ }^{13} \mathrm{CNMR}\left(75 \mathrm{MHz}, \mathrm{CDCl}_{3}\right)$ $\delta$ 136.0, 134.9, 131.9, 129.6, 129.2, 125.1, 125.1, 3.2; GCMS: 331.

1,3-Dichloro-2-(iodomethyl)benzene $2 \mathrm{j}$

White solid; m.p. $63-65^{\circ} \mathrm{C} ;{ }^{1} \mathrm{HNMR}\left(300 \mathrm{MHz}, \mathrm{CDCl}_{3}\right) \delta$ $7.30(\mathrm{~d}, J=7.8 \mathrm{~Hz}, 2 \mathrm{H}), 7.15(\mathrm{t}, J=7.8 \mathrm{~Hz}, 1 \mathrm{H}), 4.67(\mathrm{~s}, 2 \mathrm{H})$;
${ }^{13} \mathrm{C}$ NMR $\left(75 \mathrm{MHz}, \mathrm{CDCl}_{3}\right) \delta 135.1,134.7,129.2,128.5$, -0.6 ; GCMS: 286.

\section{1-(Iodomethyl)-4-isopropylbenzene $2 \mathrm{k}$}

Brown liquid; ${ }^{1} \mathrm{H}$ NMR $\left(400 \mathrm{MHz}, \mathrm{CDCl}_{3}\right) \delta 7.32$ (d, $J$ $=6.7 \mathrm{~Hz}, 2 \mathrm{H}), 7.16(\mathrm{~d}, J=6.7 \mathrm{~Hz}, 2 \mathrm{H}), 4.47(\mathrm{~s}, 2 \mathrm{H}), 2.92-$ $2.85(\mathrm{~m}, 1 \mathrm{H}), 1.24(\mathrm{~d}, J=6.9 \mathrm{~Hz}, 6 \mathrm{H}) ;{ }^{13} \mathrm{C} \mathrm{NMR}(100 \mathrm{MHz}$, $\left.\mathrm{CDCl}_{3}\right) \delta 148.7,136.5,128.7,126.9,33.8,23.8,6.1$; GCMS: 260.

2-Iodo-1-(iodomethyl)-4-methoxybenzene 21

Pale yellow solid; m.p. $58-61{ }^{\circ} \mathrm{C} ;{ }^{1} \mathrm{H}$ NMR $(400 \mathrm{MHz}$, $\left.\mathrm{CDCl}_{3}\right) \delta 7.41(\mathrm{~d}, J=2.2 \mathrm{~Hz}, 1 \mathrm{H}), 7.25(\mathrm{dd}, J=8.4,2.2 \mathrm{~Hz}$, $1 \mathrm{H}), 6.85(\mathrm{~d}, J=8.4 \mathrm{~Hz}, 1 \mathrm{H}), 4.42(\mathrm{~s}, 2 \mathrm{H}), 3.90(\mathrm{~s}, 3 \mathrm{H})$; ${ }^{13} \mathrm{C}$ NMR $\left(100 \mathrm{MHz}, \mathrm{CDCl}_{3}\right) \delta 154.6,132.3,130.5,128.2$, 122.4, 112.1, 56.2, 6.2; GCMS: 373.

Benzyl 4-(iodomethyl)phenyl ether $\mathbf{2 m}$

White solid; m.p. $79-80{ }^{\circ} \mathrm{C} ;{ }^{1} \mathrm{H} N M R\left(400 \mathrm{MHz}, \mathrm{CDCl}_{3}\right) \delta$ 7.46-7.30 (m, 7H), 6.93-6.89 (m, 2H), 5.06 (s, 2H), 4.48 (s, $2 \mathrm{H}) ;{ }^{13} \mathrm{C} \mathrm{NMR}\left(100 \mathrm{MHz}, \mathrm{CDCl}_{3}\right) \delta$ 158.4, 136.7, 131.6, 130.0, 128.6, 128.0, 127.4, 115.1, 70.0, 6.5; GCMS: 324.

\section{4-(Iodomethyl)benzoic acid 2n}

Off-white solid; m.p. $82-85^{\circ} \mathrm{C}$; ${ }^{1} \mathrm{H}$ NMR $(400 \mathrm{MHz}$, DMSO- $\left.d_{6}\right) \delta 12.98$ (br s, $\left.1 \mathrm{H}\right), 7.85$ (d, $\left.J=8.2 \mathrm{~Hz}, 2 \mathrm{H}\right)$, $7.51(\mathrm{~d}, J=8.2 \mathrm{~Hz}, 2 \mathrm{H}), 4.65$ (s, 2H); ${ }^{13} \mathrm{C}$ NMR $(100 \mathrm{MHz}$, DMSO- $\left.d_{6}\right) \delta 166.9,144.9,129.9,129.7,129.2,129.1,5.9$; LCMS: $263(\mathrm{M}+1)$.

\section{Methyl 4-(iodomethyl)benzoate 2o}

White solid; m.p. $76-78^{\circ} \mathrm{C} ;{ }^{1} \mathrm{H}$ NMR $\left(400 \mathrm{MHz}, \mathrm{CDCl}_{3}\right)$ $\delta 7.96(\mathrm{~d}, J=8.2 \mathrm{~Hz}, 2 \mathrm{H}), 7.43(\mathrm{~d}, J=8.2 \mathrm{~Hz}, 2 \mathrm{H}), 4.46(\mathrm{~s}$, 2H), 3.91 (s, $3 \mathrm{H}) ;{ }^{13} \mathrm{C}$ NMR (100 MHz, $\left.\mathrm{CDCl}_{3}\right) \delta 166.4$, 144.3, 130.0, 129.5, 128.7, 128.6, 52.1, 3.8; GCMS: 276.

1-[3-(Iodomethyl)phenyl]ethanone 2p

Off-white solid; m.p. $70-72{ }^{\circ} \mathrm{C}$; ${ }^{1} \mathrm{H}$ NMR $(400 \mathrm{MHz}$, $\left.\mathrm{CDCl}_{3}\right) \delta 7.96(\mathrm{~d}, J=1.5 \mathrm{~Hz}, 1 \mathrm{H}), 7.85-82(\mathrm{dd}, J=7.7,1.5$ $\mathrm{Hz}, 1 \mathrm{H}), 7.59(\mathrm{~d}, J=7.7 \mathrm{~Hz}, 1 \mathrm{H}), 7.42(\mathrm{t}, J=7.7 \mathrm{~Hz}, 1 \mathrm{H}), 4.49$ (s, 2H), $2.62(\mathrm{~s}, 3 \mathrm{H}) ;{ }^{13} \mathrm{C} \mathrm{NMR}\left(75 \mathrm{MHz}, \mathrm{CDCl}_{3}\right) \delta 197.4$, 139.9, 137.5, 133.2, 129.1, 128.2, 127.7, 26.6, 4.0; GCMS: 260.

\section{4-(Iodomethyl)benzonitrile 2q}

Yellow solid; m.p. $143-146^{\circ} \mathrm{C} ;{ }^{1} \mathrm{HNMR}\left(300 \mathrm{MHz}, \mathrm{CDCl}_{3}\right)$ $\delta 7.59(\mathrm{~d}, J=8.3 \mathrm{~Hz}, 2 \mathrm{H}), 7.47$ (d, $J=8.3 \mathrm{~Hz}, 2 \mathrm{H}), 4.44$ (s, $2 \mathrm{H}) ;{ }^{13} \mathrm{CNMR}\left(75 \mathrm{MHz}, \mathrm{CDCl}_{3}\right) \delta 144.6,132.5,129.3,118.3$, 111.5, 2.7; GCMS: 243.

\section{3-(Iodomethyl)benzonitrile $2 \mathbf{r}$}

Pale yellow solid; m.p. $115-117^{\circ} \mathrm{C}$; ${ }^{1} \mathrm{H}$ NMR $(400 \mathrm{MHz}$, $\left.\mathrm{CDCl}_{3}\right) \delta 7.66(\mathrm{~s}, 1 \mathrm{H}), 7.61(\mathrm{~d}, J=7.0 \mathrm{~Hz}, 1 \mathrm{H}), 7.54-7.53$ $(\mathrm{d}, J=7.0 \mathrm{~Hz}, 1 \mathrm{H}), 7.44-7.42(\mathrm{~d}, J=7.0 \mathrm{~Hz}, 1 \mathrm{H}), 4.42$ (s, $2 \mathrm{H}) ;{ }^{13} \mathrm{CNMR}\left(75 \mathrm{MHz}, \mathrm{CDCl}_{3}\right) \delta 140.8,133.0,132.0,131.2$, 129.6, 118.2, 112.8, 2.5; GCMS: 243.

\section{1-(Iodomethyl)-3-nitrobenzene $2 \mathrm{~s}$}

Yellow solid; m.p. $84-85^{\circ} \mathrm{C} ;{ }^{1} \mathrm{HNMR}\left(400 \mathrm{MHz}, \mathrm{CDCl}_{3}\right)$ 
$\delta 8.25(\mathrm{t}, J=1.9 \mathrm{~Hz}, 1 \mathrm{H}), 8.13-8.11(\mathrm{~m}, 1 \mathrm{H}), 7.71(\mathrm{~d}, J=$ $7.9,1.2 \mathrm{~Hz}, 1 \mathrm{H}), 7.50(\mathrm{t}, J=7.9 \mathrm{~Hz}, 1 \mathrm{H}), 4.51(\mathrm{~s}, 2 \mathrm{H}) ;{ }^{13} \mathrm{C}$ NMR (75 MHz, $\left.\mathrm{CDCl}_{3}\right) \delta$ 148.3, 141.3, 134.6, 129.81, 123.4, 122.6, 2.06; GCMS: 263.

\section{1-(Allyloxy)-2-(iodomethyl)benzene 2t}

Pale yellow liquid; ${ }^{1} \mathrm{H} \mathrm{NMR}\left(300 \mathrm{MHz}, \mathrm{CDCl}_{3}\right) \delta 7.33-$ $7.16(\mathrm{~m}, 2 \mathrm{H}), 6.91-6.81(\mathrm{~m}, 2 \mathrm{H}), 6.18-6.07(\mathrm{~m}, 1 \mathrm{H}), 5.42$ $(\mathrm{d}, J=17.2 \mathrm{~Hz}, 1 \mathrm{H}), 5.32(\mathrm{~d}, J=10.5 \mathrm{~Hz}, 1 \mathrm{H}), 4.65(\mathrm{~d}, J=$ $4.7 \mathrm{~Hz}, 2 \mathrm{H}), 4.53(\mathrm{~s}, 2 \mathrm{H}) ;{ }^{13} \mathrm{CNMR}\left(75 \mathrm{MHz}, \mathrm{CDCl}_{3}\right) \delta 156.1$, 133.0, 130.1, 129.4, 127.6, 120.7, 117.2, 112.1, 68.6, 1.2; GCMS: 274.

1-(Allyloxy)-4-(iodomethyl)benzene 2u

Yellow liquid; ${ }^{1} \mathrm{H}$ NMR ( $\left.300 \mathrm{MHz}, \mathrm{CDCl}_{3}\right) \delta$ 7.33-7.27 (m, 2H), 6.87-6.83 (m, 2H), 6.08-6.05 (m, 1H), 5.44 (d, J $=16.8 \mathrm{~Hz}, 1 \mathrm{H}), 5.29(\mathrm{~d}, J=10.0 \mathrm{~Hz}, 1 \mathrm{H}), 4.65(\mathrm{~d}, J=4.8 \mathrm{~Hz}$, 2H), 4.54 (s, $2 \mathrm{H}) ;{ }^{13} \mathrm{C} \mathrm{NMR}\left(75 \mathrm{MHz}, \mathrm{CDCl}_{3}\right) \delta 158.1,135.6$, 133.4, 129.9, 117.7, 115.0, 68.7, 5.5; GCMS: 274.

\section{[(1-E)-3-Iodopro-1-en-1-yl]benzene 2v}

Pale yellow solid; m.p. $58-60{ }^{\circ} \mathrm{C} ;{ }^{1} \mathrm{H}$ NMR $(300 \mathrm{MHz}$, $\left.\mathrm{CDCl}_{3}\right) \delta 7.40-7.23(\mathrm{~m}, 5 \mathrm{H}), 6.62(\mathrm{~d}, J=15.6 \mathrm{~Hz}, 1 \mathrm{H}), 6.50-$ $6.40(\mathrm{~m}, 1 \mathrm{H}), 4.14(\mathrm{~d}, J=7.9 \mathrm{~Hz}, 2 \mathrm{H}) ;{ }^{13} \mathrm{C} \mathrm{NMR}(75 \mathrm{MHz}$, $\left.\mathrm{CDCl}_{3}\right) \delta 135.8,133.0,128.5,128.1,126.8,126.5,6.7$; GCMS: 244.

\section{1-Ethynyl-4-(iodomethyl)benzene $2 w$}

Pale yellow solid; m.p. $52-53{ }^{\circ} \mathrm{C} ;{ }^{1} \mathrm{H}$ NMR $(300 \mathrm{MHz}$, $\left.\mathrm{CDCl}_{3}\right) \delta 7.42(\mathrm{~d}, J=8.2 \mathrm{~Hz}, 2 \mathrm{H}), 7.33(\mathrm{~d}, J=8.2 \mathrm{~Hz}, 2 \mathrm{H})$, $4.44(\mathrm{~s}, 2 \mathrm{H}), 3.10(\mathrm{~s}, J=1 \mathrm{H}) ;{ }^{13} \mathrm{C} \mathrm{NMR}\left(75 \mathrm{MHz}, \mathrm{CDCl}_{3}\right)$ $\delta$ 139.9, 132.4, 128.6, 121.6, 83.1, 77.8, 4.5; GCMS: 242.

\section{1-(Iodomethyl)-4-(phenylethynyl)benzene $2 x$}

Pale yellow solid; m.p. $54-56{ }^{\circ} \mathrm{C} ;{ }^{1} \mathrm{H}$ NMR $(300 \mathrm{MHz}$, $\left.\mathrm{CDCl}_{3}\right) \delta$ 7.66-7.62 (m, 2H), 7.53-7.50 (m, 1H), 7.46-7.39 (m, 5H), 7.37-7.25 (m, 1H), $4.71(\mathrm{~s}, 2 \mathrm{H}) ;{ }^{13} \mathrm{CNMR}(75 \mathrm{MHz}$, $\left.\mathrm{CDCl}_{3}\right) \delta 140.5,132.5,131.5,128.9,128.7,128.5,128.4$, 128.1, 127.9, 125.6, 123.1, 122.7, 95.8, 86.6, 4.4; GCMS: 318.

\section{RESULTS AND DISCUSSION}

To execute this task, $p$-bromobenzaldehyde 1a, a model substrate shown in Scheme 1, was stirred with $\mathrm{Et}_{3} \mathrm{SiH}$, $\mathrm{TfOH}$ and $\mathrm{NaI}$ as an iodide source in acetonitrile at room temperature for $2 \mathrm{~h}$ furnished $\mathbf{2 a}$ in $75 \%$ yield (Table 1). To further optimize conditions, the reaction was conducted

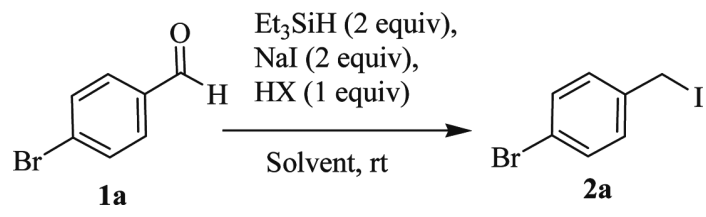

Scheme 1. Reductive iodination of $p$-bromobenzaldehyde.
Table 1. Optimization of reductive iodination condition with various solvents and protic acids ${ }^{\mathrm{a}}$

\begin{tabular}{ccccc}
\hline Entry & Acids & Solvents & Time $(\mathrm{h})$ & Yield $^{\mathrm{b}}(\%)$ \\
\hline 1 & $\mathrm{TfOH}$ & $\mathrm{CH}_{3} \mathrm{CN}$ & 12 & 75 \\
2 & $\mathrm{TfOH}$ & DME & 12 & 35 \\
3 & $\mathrm{TfOH}$ & Toluene & 12 & 15 \\
4 & $\mathrm{TfOH}$ & $\mathrm{THF}$ & 12 & trace \\
5 & $\mathrm{TfOH}$ & $\mathrm{DMF}$ & 12 & - \\
6 & $\mathrm{TfOH}$ & $\mathrm{DCM}$ & 12 & trace \\
7 & $\mathrm{CH}_{3} \mathrm{COOH}$ & $\mathrm{CH}_{3} \mathrm{CN}$ & 12 & - \\
8 & $\mathrm{TFA}$ & $\mathrm{CH}_{3} \mathrm{CN}$ & 12 & - \\
9 & $\mathrm{MeSO}_{3} \mathrm{H}$ & $\mathrm{CH}_{3} \mathrm{CN}$ & 12 & trace \\
10 & $\mathrm{TfOH}$ & $\mathrm{CH}_{3} \mathrm{CN} / \mathrm{DME}$ & 1 & 90 \\
\hline
\end{tabular}

${ }^{\text {aReactions were performed on } p \text {-bromobenzaldehyde }(1 \mathrm{mmol}) \text { in }}$ $\mathrm{CH}_{3} \mathrm{CN}(5 \mathrm{~mL})$ with 2 equiv. of triethylsilane $\left(\mathrm{Et}_{3} \mathrm{SiH}\right), 12$ equiv. of acid, 2 equiv. of NaI. ' Isolated yield. DME: 1,2-dimethoxyethane.

by changing the solvents, Brønsted acids and reaction time. Solvents such as DME and toluene did not improve the yield and formation of the desired product was not observed when the reaction was carried out in THF, DMF and DCM (entry 2-6). Similar results were obtained when the reaction was conducted in the presence of various protic acids. Surprisingly, using the mixture of acetonitrile/DME (8:2) with TfOH resulted in complete consumption of $1 \mathrm{a}$ in $1 \mathrm{~h}$ and delivered 2a in high yield (90\%) (Table 1). ${ }^{9 a, b}$ Additionally, $n \mathrm{Bu}_{4} \mathrm{NI}$ and $n \mathrm{Me}_{4} \mathrm{NI}$ were used as iodide source instead of sodium iodide under the optimized condition (entry 10) showed complete decomposition.

In order to explore the application of this protocol, we employed the optimized conditions on benzaldehydes bearing various functional groups. Bromo, chloro, fluoro substituted benzaldehydes $\mathbf{1} \mathbf{b}-\mathbf{1} \mathbf{j}$ underwent smooth reductive iodination to deliver the corresponding benzyl iodides $\mathbf{2 b}-\mathbf{2 j}$ in high yield (Table 2). Similarly, alkyl and alkoxy substituted benzaldehydes gave the desired products $\mathbf{2 k} \mathbf{k} \mathbf{2 m}$. Carbonyl derivatives such as acid and ester group bearing benzaldehydes led to the corresponding benzyl iodides $\mathbf{2 n - 2 o}$ in high yield. The desired benzyl iodide $\mathbf{2 p}$ was obtained in high yield without affecting the keto functionality. Nitro and cyano substituted benzaldehydes also delivered the desired products $\mathbf{2 q} \mathbf{- 2} \mathbf{s}$ as shown in the Table 2 .

To further demonstrate the efficiency of this protocol, allyl substituted benzaldehydes were converted into corresponding the benzyl iodide derivatives $2 \mathbf{t}-\mathbf{2} \mathbf{u}$. Cinnamaldehyde led to the iodo derivative $2 \mathbf{v}$ wherein the double bond is preserved. Triple bonds also remained unaffected when terminal and internal alkyne substituted benzaldehydes were subjected to the reductive iodination to afford $\mathbf{2 w}-\mathbf{2 x}$ (Table 2). However, aliphatic aldehydes fail to 
Table 2. Synthesis of various benzyl iodides through a reductive iodination

\begin{tabular}{|c|c|c|c|c|c|}
\hline Entry & Aldehyde & Product & Time (min) & Yield $^{\mathrm{a}}(\%)$ & $\mathrm{Mp}\left({ }^{\circ} \mathrm{C}\right) /($ lit. $)$ \\
\hline 1 & $4-\mathrm{BrC}_{6} \mathrm{H}_{4} \mathrm{CHO}$ & $2 \mathbf{2 a}$ & 60 & 90 & $71-73$ \\
\hline 2 & 2- $\mathrm{BrC}_{6} \mathrm{H}_{4} \mathrm{CHO}$ & $2 \mathbf{b}$ & 30 & 90 & $58-60$ \\
\hline 3 & $3-\mathrm{BrC}_{6} \mathrm{H}_{4} \mathrm{CHO}$ & $2 \mathrm{c}$ & 45 & 89 & $50-55$ \\
\hline 4 & 4- $\mathrm{ClC}_{6} \mathrm{H}_{4} \mathrm{CHO}$ & 2d & 60 & 87 & $62-64$ \\
\hline 5 & $2-\mathrm{FC}_{6} \mathrm{H}_{4} \mathrm{CHO}$ & $2 \mathrm{e}$ & 45 & 88 & \\
\hline 6 & 2-Cl,4- $\mathrm{FC}_{6} \mathrm{H}_{3} \mathrm{CHO}$ & $2 f$ & 45 & 85 & $50-52$ \\
\hline 7 & $3-\mathrm{Br}, 2-\mathrm{FC}_{6} \mathrm{H}_{3} \mathrm{CHO}$ & $2 \mathrm{~g}$ & 60 & 84 & $54-56$ \\
\hline 8 & $5-\mathrm{Br}, 2-\mathrm{OMeC}{ }_{6} \mathrm{H}_{3} \mathrm{CHO}$ & $2 \mathrm{~h}$ & 50 & 83 & $55-58$ \\
\hline 9 & $2-\mathrm{Br}, 6-\mathrm{ClC}_{6} \mathrm{H}_{3} \mathrm{CHO}$ & $2 \mathbf{i}$ & 60 & 86 & $75-78$ \\
\hline 10 & 2-Cl,6- $\mathrm{ClC}_{6} \mathrm{H}_{3} \mathrm{CHO}$ & $2 \mathrm{j}$ & 60 & 90 & $63-65$ \\
\hline 11 & 4-i $\mathrm{PrC}_{6} \mathrm{H}_{4} \mathrm{CHO}$ & $2 \mathbf{k}$ & 45 & 87 & \\
\hline 12 & 2-I,4- $\mathrm{OMeC}_{6} \mathrm{H}_{3} \mathrm{CHO}$ & 21 & 60 & 78 & $58-61$ \\
\hline 13 & $4-\mathrm{OBnC}_{6} \mathrm{H}_{4} \mathrm{CHO}$ & $2 m$ & 35 & 75 & $79-80$ \\
\hline 14 & 4- $\mathrm{COOHC}_{6} \mathrm{H}_{4} \mathrm{CHO}$ & $2 n$ & 30 & 85 & $82-85$ \\
\hline 15 & $4-\mathrm{COOCH}_{3} \mathrm{C}_{6} \mathrm{H}_{4} \mathrm{CHO}$ & 20 & 35 & 87 & $76-78$ \\
\hline 16 & $3-\mathrm{COCH}_{3} \mathrm{C}_{6} \mathrm{H}_{4} \mathrm{CHO}$ & $2 p$ & 45 & 88 & $70-72$ \\
\hline 17 & 4- $\mathrm{CNC}_{6} \mathrm{H}_{4} \mathrm{CHO}$ & $2 q$ & 50 & 73 & $143-146$ \\
\hline 18 & $3-\mathrm{CNC}_{6} \mathrm{H}_{4} \mathrm{CHO}$ & $2 \mathbf{r}$ & 38 & 75 & $115-117$ \\
\hline 19 & $3-\mathrm{O}_{2} \mathrm{NC}_{6} \mathrm{H}_{4} \mathrm{CHO}$ & $2 s$ & 45 & 76 & $84-85$ \\
\hline 20 & 2-AllyloxyC $\mathrm{C}_{6} \mathrm{H}_{4} \mathrm{CHO}$ & $2 t$ & 50 & 78 & \\
\hline 21 & 4-AllyloxyC $\mathrm{C}_{6} \mathrm{H}_{4} \mathrm{CHO}$ & $2 u$ & 38 & 76 & $58-60$ \\
\hline 22 & Cinnamaldehyde & $2 \mathbf{v}$ & 50 & 82 & $52-53$ \\
\hline 23 & 4-Ethynylbenzaldehyde & $2 w$ & 30 & 72 & $54-56$ \\
\hline 24 & 4-(Phenylethynyl)benzaldehyde & $2 x$ & 40 & 81 & $152-153$ \\
\hline
\end{tabular}

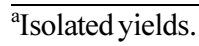

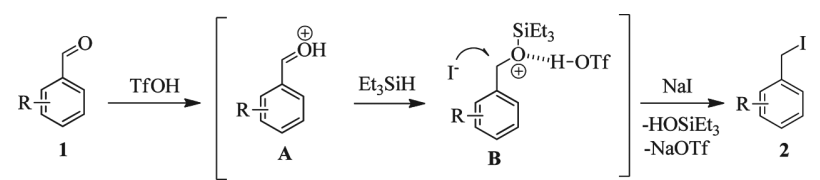

Scheme 2. Tentative mechanism for reductive iodination.

undergo reductive iodination.

A tentative mechanism has been shown in Scheme 2 for the reductive iodination. At first, the aldehyde 1 gets protonated to form a protonated aldehyde $\mathbf{A}$ which undergoes reduction under $\mathrm{Et}_{3} \mathrm{SiH}$ to generate $\mathbf{B}$. Nucleophilic displacement on $\mathbf{B}$ with iodide gives benzyl iodide 2 .

\section{CONCLUSION}

In conclusion, we have developed a simple and efficient protocol for the preparation of benzyl iodides from aryl aldehydes in a single step through a reductive iodination protocol. This transformation was performed under ambient condition using a combination $\mathrm{Et}_{3} \mathrm{SiH}, \mathrm{CF}_{3} \mathrm{SO}_{3} \mathrm{H}$ and NaI. Also, it exhibits broad functional group tolerance.

Acknowledgments. The publication cost of this paper was supported by the Korean Chemical Society.

\section{REFERENCES}

1. (a) Reinhard, R.; Schmidt, B. F. J. Org. Chem. 1998, 63, 2434-2441. (b) Smith, T. H.; Fujiwara, A. N.; Henry, D. W. J. Med. Chem. 1979, 22, 40.

2. Loksha, Y. M.; El-Badawi, M. A.; El-Barbary, A. A.; Pedersen, E. B.; Nielsen, C. Arch. Pharm. 2003, 336, 175.

3. Andrus, M. B.; Hicken, E. J.; Stephens, J. C.; Bedke, D. K. J. Org. Chem. 2005, 70, 9470.

4. DeGraw, J. I.; Colwell, W. T.; Crase, J.; Smith, R. L.; Piper, J. 
R.; Waud, W. R.; Sirotnak, F. M. J. Med. Chem. 1997, 40, 370 .

5. (a) Vankar, Y. D.; Rao, C. T. Tetrahedron Lett. 1985, 26, 2717. (b) Haynes, R. K.; Holden, M. Aust. J. Chem. 1982, 35, 517. (c) Di Deo, M.; Marcantoni, E.; Torregiani, E.; Bartoli, G.; Bellucci, M. C.; Bosco, M.; Sambri, L. J. Org. Chem. 2000, 65, 2830. (d) Joseph, R.; Pallan, P.; Sudalai, A.; Ravindranathan, T. Tetrahedron Lett. 1995, 36, 609. (e) Tajbakhsh, M.; Hosseinzadeh, R.; Lasemi, Z. Synlett 2004, 635. (f) Iranpoor, N.; Firouzabadi, H.; Aghapour, A.; Vaezzadeh, A. R. Tetrahedron 2002, 58, 8689. (g) Firouzabadi, H.; Iranpoor, N.; Jafarpour, M. Tetrahedron Lett. 2004, 45, 7451. (h) Falck, J. R.; Manna, S. Synth. Commun. 1985, 15, 663. (i) Bhor, M. D.; Panda, A. G.; Nandurkar, N. S.; Bhanage, B. M. Tetrahedron Lett. 2008, 49, 6475.

6. (a) Aizpurua, J. M.; Lecea, B.; Palomo, C. Tetrahedron Lett. 1984, 25, 1103. (b) Aizpurua, J. M.; Lecea, B.; Palomo, C. Can. J. Chem. 1986, 64, 2342. (c) Li, Z.; Sheng, C.; Yang, C.; Qiu, H. Org. Prep. Proc. Int. 2007, 39, 608. (d) Keinan,
E.; Perez, D.; Sahai, M.; Shvily, R. J. Org. Chem. 1990, 55, 2927. (e) Lecea, B.; Azipurua, J. M.; Palomo, C. Tetrahedron 1985, 41, 4657. (f) Das, B.; Srinivas, Y.; Holla, H.; Laxminarayana, K.; Narender, R. Tetrahedron Lett. 2007, $48,6681$.

7. (a) Michael, P. D.; Donald, J. D.; Stephen, J. D.; Dale, A. K.; Abayomi, A. O.; Charles, T. W.; Steven, M. Z. J. Org. Chem. 1974, 39, 2740. (b) Evans, D. V.; Fitch, D. M.; Smith, T. E.; Cee, V. J. J. Am. Chem. Soc. 2000, 122, 10033. (c) Lam, H. W.; Joensuu, P. M. A. Org. Lett. 2005, 7, 4225.

8. (a) Chang, Y. H.; Pinnick, H. W. J. Org. Chem. 1978, 43, 374. (b) Aghayan, M. M.; Boukherroub, R.; Rahimifard, M. J. Organomet. Chem. 2008, 693, 3567.

9. (a) In our hands, lower conversion was observed when TfOH was used in catalytic amount $(25 \mathrm{~mol} \%)$ whereas complete conversion was seen with 1 equiv. of TfOH under the reaction condition; (b) Similarly, no product formation was not observed under Lewis acid condition. 